Article

\title{
Quantum Correlation via Skew Information and Bell Function Beyond Entanglement in a Two-Qubit Heisenberg XYZ Model: Effect of the Phase Damping
}

\author{
Abdel-Baset A. Mohamed ${ }^{1,2, *(\mathbb{D}}$, Ahmed Farouk ${ }^{3}\left(\mathbb{D}\right.$, Mansour F. Yassen $^{1}(\mathbb{D}$ and \\ Hichem Eleuch 4,5 (D) \\ 1 Department of Mathematics, College of Science and Humanities in Al-Aflaj, Prince Sattam bin \\ Abdulaziz University, Al-Aflaj 11942, Saudi Arabia; mf.ali@psau.edu.sa \\ 2 Faculty of Science, Assiut University, Assiut 71515, Egypt \\ 3 Department of Physics and Computer Science, Faculty of Science, Wilfrid Laurier University, Waterloo, \\ ON N2L 3C5, Canada; afarouk@wlu.ca \\ 4 Institute for Quantum Science and Engineering, Texas A\&M University, College Station, TX 77843, USA; \\ hichem.eleuch@adu.ac.ae \\ 5 Department of Applied Sciences and Mathematics, College of Arts and Sciences, Abu Dhabi University, \\ Abu Dhabi 59911, UAE \\ * Correspondence: a.mohamed@psau.edu.sa
}

Received: 14 May 2020; Accepted: 25 May 2020; Published: 29 May 2020

\begin{abstract}
In this paper, we analyze the dynamics of non-local correlations (NLCs) in an anisotropic two-qubit Heisenberg XYZ model under the effect of the phase damping. An analytical solution is obtained by applying a method based on the eigenstates and the eigenvalues of the Hamiltonian. It is observed that the generated NLCs are controlled by the Dzyaloshinskii-Moriya interaction, the purity indicator, the interaction with the environment, and the anisotropy. Furthermore, it is found that the quantum correlations, as well as the sudden death and sudden birth phenomena, depend on the considered physical parameters. In particular, the system presents a special correlation: the skew-information correlation. The log-negativity and the uncertainty-induced non-locality exhibit the sudden-change behavior. The purity of the initial states plays a crucial role on the generated nonlocal correlations. These correlations are sensitive to the DM interaction, anisotropy, and phase damping.
\end{abstract}

Keywords: two-qubit; Heisenberg spin chain; phase damping; skew-information correlation

\section{Introduction}

The recent growth of the quantum technology has promulgated the preservation of the quantum correlation to become a central problem [1-5]. The investigations of the non-local correlations (NLCs) (including measurement-induced disturbance [6], quantum discord [7,8], and the geometrical correlations of measurement-induced nonlocality [9] and skew information theory [10]) become the primary roots of the quantum information [1]. The definitions of NLC measures were mainly on the Hilbert-Schmidt norm (2-norm), the skew information theory, and trace norm [11]. These definitions were exalting new types of the NLCs beyond the quantum entanglement (QE), which have generated many investigations $[12,13]$.

It was proven that the NLC quantifiers, which, based on the 2-norm, are not proper measures for the NLC, because they encounter the local ancilla problem [14] in which the NLC quantifiers change arbitrarily under local operations on an unmeasured ancilla state. Therefore, two NLC quantifiers (local quantum uncertainty (LQU) [15] and uncertainty-induced quantum nonlocality (UIN) [16]) are 
recently defined based on the skew information theory [10]. LQU and UIN are used to quantify the minimal and maximal skew information correlations, respectively. If the LQU and UIN are equal, they present the same correlation that is called "skew-information correlation" [12].

Loss of the non-local correlations resulting from the coupling of a two-qubit system to its environment transforms a correlated two-qubit state into a statistical mixture state [17]. As the coupling of a two-qubit system to its environment leads to different types of decoherence as qubit energy and the phase damping. In the phase-damping model, the phase is damped without energy relaxation [18]. The phase-damping prevents or minimizes the effect of the environmental noise in the practical realization of quantum information processes [19].

Previous studies of the NLCs in a two-qubit Heisenberg XYZ chain were limited to the quantum entanglement (QE) and quantum memory-assisted entropic uncertainty relation [20,21]. Solid-state quantum systems are the most natural candidates for the qubit based on quantum dots, electronic spin, and optical lattices [22,23].

Here, we investigate the non-local correlations for two-qubit Heisenberg XYZ chain that includes a virtual spin Dzyaloshinskii-Moriya interaction, which results from the Anderson's superexchange interaction. Our study is conducted with new NLC quantifiers based on the skew information theory (LQU and UIN) and Bell's inequality. Therefore, an analytical solution of the two-qubit Heisenberg XYZ model will be found by applying a new method based on the eigenstates and the eigenvalues of the Hamiltonian. Our results show that is possible to control the generated NLCs, the skew information correlation, the Bell's inequality correlation, and the sudden death/brith. The type of initial state manipulates the unitary interaction between the two qubits.

In this contribution, the physical model and its density matrix, are introduced in Section 2. NLC functions will be presented in Section 3. The discussions of the non-local correlation dynamics is conferred in Section 4. Finally, we present our conclusions in Section 5.

\section{The Physical Model and Its Master Equation}

The incorporating Hamiltonian of the Heisenberg XYZ chain with Dyzaloshinskii-Moriya (DM) interaction is given by

$$
\hat{H}=\sum_{k=1}^{n}\left[J_{x} \hat{\sigma}_{k}^{x} \hat{\sigma}_{k+1}^{x}+J_{y} \hat{\sigma}_{k}^{y} \hat{\sigma}_{k+1}^{y}+J_{z} \hat{\sigma}_{k}^{z} \hat{\sigma}_{k+1}^{z}+\vec{D} \cdot\left(\vec{\sigma}_{k} \times \vec{\sigma}_{k+1}\right)\right]
$$

where $J_{i}(i=x, y, z)$ are the symmetric spin-spin interaction coupling constants, $\vec{\sigma}_{k}$ is the Pauli operators vector for any qubit, and $\vec{D}$ is the DM vector. Despite its simplicity, the model describes the magnetism of several real physical systems based on the DM interaction which arises from spin-orbit coupling [24,25]. Moreover, it was successful in explaining the ferromagnetic and antiferromagnetic behaviors. Besides, the models of the $\mathrm{XYZ}$ chain have been used to accomplish more important resources of the quantum information, like quantum coherence [26] and quantum correlations [27-29]. According to experimental demonstration [30], the system of spin- $\frac{1}{2}$ pyrochlores $\mathrm{R}_{2} \mathrm{~V}_{2} \mathrm{O}_{7}$ can realize the $\mathrm{DM}$ interaction in the anisotropic $X Y Z$ chain. Here, we consider the case of the two-qubit of the spin chain with the DM interaction along z-axis. Thus, the Hamiltonian can be expressed as

$$
\hat{H}=J_{x} \hat{\sigma}_{1}^{x} \hat{\sigma}_{2}^{x}+J_{y} \hat{\sigma}_{1}^{y} \hat{\sigma}_{2}^{y}+J_{z} \hat{\sigma}_{1}^{z} \hat{\sigma}_{2}^{z}+\chi_{z}\left(\hat{\sigma}_{1}^{x} \hat{\sigma}_{2}^{y}-\hat{\sigma}_{1}^{y} \hat{\sigma}_{2}^{x}\right),
$$

where $\chi_{z}$ represents the strength of DM interaction. This Hamiltonian is exactly diagonalizable and satisfies the eigenvalue problem: $\hat{H}\left|\Psi_{k}\right\rangle=E_{k}\left|\Psi_{k}\right\rangle(k=1-4)$, with four eigenstates $\left|\Psi_{k}\right\rangle$ corresponds to four eigenvalues $E_{k}$. In the basis two-qubit states: $\left\{\left|\omega_{1}\right\rangle=\left|0_{A} 0_{B}\right\rangle,\left|\omega_{2}\right\rangle=\left|0_{A} 1_{B}\right\rangle,\left|\omega_{3}\right\rangle=\left|1_{A} 0_{B}\right\rangle\right.$, $\left.\left|\omega_{4}\right\rangle=\left|1_{A} 1_{B}\right\rangle\right\}$, the eigenstates $\left|\Psi_{k}\right\rangle$ of Equation (1) are given by 


$$
\left(\begin{array}{l}
\left|\Psi_{1}\right\rangle \\
\left|\Psi_{2}\right\rangle \\
\left|\Psi_{3}\right\rangle \\
\left|\Psi_{4}\right\rangle
\end{array}\right)=M\left(\begin{array}{l}
\left|\omega_{1}\right\rangle \\
\left|\omega_{2}\right\rangle \\
\left|\omega_{3}\right\rangle \\
\left|\omega_{4}\right\rangle
\end{array}\right), M=\left(\begin{array}{cccc}
\frac{1}{\sqrt{2}} & 0 & 0 & \frac{1}{\sqrt{2}} \\
0 & \frac{1}{\sqrt{2}} & \frac{\mu}{\sqrt{2}} & 0 \\
0 & \frac{1}{\sqrt{2}} & -\frac{\mu}{\sqrt{2}} & 0 \\
\frac{1}{\sqrt{2}} & 0 & 0 & -\frac{1}{\sqrt{2}}
\end{array}\right)
$$

and the corresponding eigenvalues are

$$
\begin{aligned}
& E_{1}=J_{x}-J_{y}+J_{z}, \quad E_{2}=J_{z}+\sqrt{4 \chi_{z}^{2}+S^{2}} \\
& E_{3}=J_{z}-\sqrt{4 \chi_{z}^{2}+S^{2}}, \quad E_{4}=-J_{x}+J_{y}+J_{z}
\end{aligned}
$$

with $\mu=\left(S+2 i \chi_{z}\right) / \sqrt{4 \chi_{z}^{2}+S^{2}}=u+i v$ and $S=J_{x}+J_{y}$.

The principal purpose here is to investigate the robustness of the generated non-local correlations due to the unitary two-qubit interaction and against the effects of the phase damping, in the phase-damping model. In this model, (1) the phase is damped without energy relaxation [18,31,32] and (2) the reservoir is coupled to the two-qubit systems via $\sigma_{i}^{+}=\left|1_{i}\right\rangle\left\langle 0_{i}\right|$ and $\sigma_{i}^{-}=\left|0_{i}\right\rangle\left\langle 1_{i}\right|(i=A, B)$. The master equation of the model is given by

$$
\begin{aligned}
\frac{d \hat{\rho}(t)}{d t} & =-i[\hat{H}, \hat{\rho}(t)] \\
& +\frac{1}{2} \sum_{i=A, B} \gamma_{i}\left[2\left|1_{i}\right\rangle\left\langle 1_{i}|\hat{\rho}(t)| 1_{i}\right\rangle\left\langle 1_{i}|-| 1_{i}\right\rangle\left\langle 1_{i}|\hat{\rho}(t)-\hat{\rho}(t)| 1_{i}\right\rangle\left\langle 1_{i}\right|\right]
\end{aligned}
$$

where $\gamma_{i}$ are the phase damping rates, we take $\gamma_{i}=\gamma$.

To find analytical solutions for the master equation of Equation (5), we use the eigenstates representation (ESR) method, that is based on the eigenstates and the eigenvalues of the Hamiltonian of Equation (1). In ESR method, we used the canonical transform: $W(t)=e^{i \hat{H} t} \hat{\rho}(t) e^{-i \hat{H} t}$, the master equation of Equation (5) becomes

$$
\dot{W}=\frac{\gamma}{2} \sum_{i=A, B} e^{i \hat{H} t}\left[2\left|1_{i}\right\rangle\left\langle 1_{i}|\hat{\rho}(t)| 1_{i}\right\rangle\left\langle 1_{i}|-| 1_{i}\right\rangle\left\langle 1_{i}|\hat{\rho}(t)-\hat{\rho}(t)| 1_{i}\right\rangle\left\langle 1_{i}\right|\right] e^{-i \hat{H} t} .
$$

Then, we write the two-qubit operators in Equation (6) in terms ESR. Consequently, the Equation (6) can be written as

$$
\begin{aligned}
\frac{d W}{d t}= & \frac{\gamma}{2}\left\{\left|\Psi_{1}\right\rangle\left\langle\Psi_{1}|W| \Psi_{1}\right\rangle\left\langle\Psi_{1}|+| \Psi_{1}\right\rangle\left\langle\Psi_{4}|W| \Psi_{4}\right\rangle\left\langle\Psi_{1}|+| \Psi_{4}\right\rangle\left\langle\Psi_{1}|W| \Psi_{1}\right\rangle\left\langle\Psi_{4}\right|\right. \\
& \left.+\left|\Psi_{4}\right\rangle\left\langle\Psi_{4}|W| \Psi_{4}\right\rangle\left\langle\Psi_{4}|+| \Psi_{2}\right\rangle\left\langle\Psi_{2}|W| \Psi_{2}\right\rangle\left\langle\Psi_{2}|+| \Psi_{3}\right\rangle\left\langle\Psi_{3}|W| \Psi_{3}\right\rangle\left\langle\Psi_{3}\right|\right] \\
& +\left|\Psi_{3}\right\rangle\left\langle\Psi_{2}|W| \Psi_{2}\right\rangle\left\langle\Psi_{3}|+| \Psi_{2}\right\rangle\left\langle\Psi_{3}|W| \Psi_{3}\right\rangle\left\langle\Psi_{2}\right| \\
& -\sum_{n=1}^{4}\left[W\left|\Psi_{n}\right\rangle\left\langle\Psi_{n}|+| \Psi_{n}\right\rangle\left\langle\Psi_{n}\right| W\right\} .
\end{aligned}
$$

We assume that the two qubits are initially started with the important class of mixed Werner state:

$$
\begin{aligned}
\rho^{A B}(0)= & p|\varphi\rangle\left\langle\varphi|+(1-p)| 1_{A} 1_{B}\right\rangle\left\langle 1_{A} 1_{B}\right|, \\
= & \frac{p}{2}\left[(1+u)\left|\Psi_{2}\right\rangle\left\langle\Psi_{2}|+i v| \Psi_{3}\right\rangle\left\langle\Psi_{2}|-i v| \Psi_{2}\right\rangle\left\langle\Psi_{3}|+(1-u)| \Psi_{3}\right\rangle\left\langle\Psi_{3}\right|\right] \\
& +\frac{(1-p)}{2}\left[\left|\Psi_{1}\right\rangle\left\langle\Psi_{1}|-| \Psi_{4}\right\rangle\left\langle\Psi_{1}|-| \Psi_{1}\right\rangle\left\langle\Psi_{4}|+| \Psi_{4}\right\rangle\left\langle\Psi_{4}\right|\right],
\end{aligned}
$$


where $0 \leq p \leq 1$ is the purity indicator of the initial two-qubit states. The state $|\varphi\rangle$ is taken to describes the maximally correlated non-symmetric Bell state: $|\varphi\rangle=\frac{1}{\sqrt{2}}[|10\rangle+|01\rangle]$, that is not the eigenstate Hamiltonian of Equation (1). With the chosen class of the Werner state of Equation (13), we can study the generation of the NLCs when the system starts with uncorrected states, and investigate the robustness of the initial NLCs against the interactions. The Werner states have manageable features that they are robust against nonlocal loss [33]. The generation of Werner states and maximally entangled mixed states are introduced by several universal sources $[34,35]$

The elements of the density matrix $W$ of the case of $i=j, W_{i}(t)=\left\langle\Psi_{i}|W(t)| \Psi_{i}\right\rangle$, are calculated by solving the simple differential equations:

$$
\dot{W}_{1(4)}(t)=-\frac{\gamma}{2} W_{1(4)}(t)+\frac{\gamma}{2} W_{4(1)}(t), \quad \dot{W}_{2(3)}(t)=-\frac{\gamma}{2} W_{2(3)}(t)+\frac{\gamma}{2} W_{3(2)}(t),
$$

whereas in the the case of $i \neq j, W_{i j}(t)=\left\langle\Psi_{i}|W(t)| \Psi_{j}\right\rangle$, are given by

$$
W_{i j}(t)=e^{-\frac{\gamma}{2} t} W_{i j}(0) .
$$

By using Equations (13)-(15), the density matrix of the two qubits $\rho^{A B}(t)$ in the basis two-qubit states: $\left\{\left|\omega_{i}\right\rangle\right\}$, is given by

$$
\rho^{A B}(t)=\sum_{i, j=1}^{4} \rho_{i j}(t)\left|\omega_{i}\right\rangle\left\langle\omega_{j}\right|,
$$

the non-vanishing elements of $\rho_{i j}(t)$ are

$$
\begin{aligned}
\rho_{11}(t) & =\frac{1}{2}(1-p)\left(1-e^{-\frac{1}{2} \gamma t} \cos 2 \Delta t\right), \\
\rho_{14}(t) & =\left(\rho_{41}(t)\right)^{*}=-i \frac{1}{2}(1-p) e^{-\frac{1}{2} \gamma t} \sin 2 \Delta t \\
\rho_{44}(t) & =\frac{1}{2}(1-p)\left(1+e^{-\frac{1}{2} \gamma t} \cos 2 \Delta t\right), \\
\rho_{22}(t) & =\frac{p}{2}-\frac{p v}{2} e^{-\frac{1}{2} \gamma t} \sin \beta t \\
\rho_{33}(t) & =\frac{p}{2}+\frac{p v}{2} e^{-\frac{1}{2} \gamma t} \sin \beta t \\
\rho_{23}(t) & =\left(\rho_{32}(t)\right)^{*}=\frac{p \mu}{2} e^{-\frac{1}{2} \gamma t}[u+i v \cos \beta t] .
\end{aligned}
$$

$\beta=2 \sqrt{4 \chi_{z}^{2}+S^{2}}$, and the difference $\Delta=J_{x}-J_{y}$ stands for the anisotropy parameter.

The analytical solution of the density matrix of the two qubits will be used to investigate the different NLCs.

\section{NLC Functions}

Here, we study the skew information, LQU, UIN, and the Bell's inequality to explore the non-classical correlations. Moreover, these measures will be compared with the log-negativity (LN) that quantifies the entanglement [36]. For a bipartite system $\rho^{A B}(t)$, it is given by [36]

$$
N(t)=\log _{2}[1+2 n(t)],
$$

where $n(t)$ is the negativity which is defined as the absolute sum of the negative eigenvalues of the partial transpose matrix $\left(\rho^{A B}(t)\right)^{T_{A}}$ with respect to subsystem $A$. Where the elements of $\left(\rho^{A B}(t)\right)^{T_{A}}$ are

$$
\left\langle i, j\left|\left(\rho^{A B}(t)\right)^{T_{A}}\right| m, n\right\rangle=\left\langle m, j\left|\rho^{A B}(t)\right| i, n\right\rangle,
$$


where the negativity and the log-negativity are zero if and only if the considered state is separable. They are used to measure the entanglement for any two-qubit state.

\subsection{Skew Information Functions}

For a bipartite quantum $\rho^{A B}(t)$ and local observable $K$ operators, the skew information (SI) is given by

$$
I\left(\rho^{A B}(t), K\right)=-\frac{1}{2} \operatorname{Tr}\left\{\left[\sqrt{\rho^{A B}(t)}, K\right]^{2}\right\},
$$

According to this SI, other NLC measures were introduced beyond the quantum discord and the entanglement [15].

The first one is the local quantum uncertainty (LQU), which is a reliable quantifier in bipartite quantum systems [15]. LQU is defined using the SI as

$$
L\left(\rho^{A B}(t)\right)=\min _{K}\left\{I\left(\rho^{A B}(t), K\right)\right\},
$$

where the minimum is taken over all of the local operators $K$. The LQU can be considered as a measure of A discord-like quantum correlation. Compared to the quantum discord, LQU is easy to calculate and has other properties as (i) it is invariant and does not increase under the local unitary operations, and (ii) in the pure states, the LQU measures reduces to the entanglement linear entropy [15]. The closed-form of the LQU is given by

$$
L(t)=1-\lambda_{\max }\left(T_{A B}\right),
$$

where $\lambda_{\max }$ is the maximum eigenvalue of $3 \times 3$-matrix $T_{A B}=\left[t_{i j}\right]$,

$$
t_{i j}=\operatorname{Tr}\left\{\sqrt{\rho^{A B}(t)}\left(\sigma_{i} \otimes I\right) \sqrt{\rho^{A B}(t)}\left(\sigma_{j} \otimes I\right)\right\} .
$$

The second measure is the uncertainty-induced non-locality (UIN), which is defined by [16]

$$
U\left(\rho^{A B}(t)\right)=\max _{K} I\left(\rho^{A B}(t), K\right),
$$

where the maximum is taken over all of the local operators $K$, consequently, UIN is the maximal skew information between the given state and the local commuting observable $K$. The closed form of UIN for the bipartite state $\rho^{A B}(t)$ can be written as [16]

$$
U(t)= \begin{cases}1-\lambda_{\min }\left(T_{A B}\right), & \overrightarrow{\mathbf{r}}=0 \\ 1-\frac{1}{\|\overrightarrow{\mathbf{r}}\|^{2}} \overrightarrow{\mathbf{r}} T_{A B} \overrightarrow{\mathbf{r}}^{T}, & \overrightarrow{\mathbf{r}} \neq 0 .\end{cases}
$$

where $\overrightarrow{\mathbf{r}}=\left[r_{i}\right]$ is the Bloch vector of the reduced density matrix $\rho^{A B}(t)$, where $r_{i}=\operatorname{Tr}\left(\rho^{A B}(t)\left(\sigma_{i} \otimes I\right)\right)$ [17].

\subsection{Bell Function (Bf)}

The maximal violation of the Bell's inequality, $(B(t)>2)$, is used as an indicator to the NCC. The closed form of the maximal Bell's function (MBF) $B(t)$ [37] for a bipartite quantum system $\rho^{A B}(t)$ is

$$
B(t)=4 \sqrt{\xi_{1}+\xi_{2}}
$$

where $\xi_{i}(i=1,2)$ are the two largest eigenvalues of the combined matrix $R^{\dagger} R$, where $R=\left[r_{m n}\right]$ represents the correlation matrix [37], where its components are determined by $r_{m n}=\operatorname{Tr}\left\{\rho^{A B}(t)\left(\sigma_{m} \otimes \sigma_{n}\right)\right\}$. 


\section{Dynamics of NLC Functions}

Our investigations are based on the effect of the anisotropy parameter $\Delta=J_{x}-J_{y}$, the coupling to the of DM interaction $\chi_{z}$, and the coupling environment $\gamma$. The anisotropy parameter is the difference between the two spin-spin coupling constants of the $x$ and $y$ directions. In Figure 1, we show the 3D-evolution of the NLC measures $L(t), U(t), B(t)$, and $N(t)$, where $p \in[0,1]$ and $t \in[0,3]$ for the DM interaction coupling $\chi_{z}=0.5$, the anisotropic parameter $\Delta=1.5$, and $J_{x}=2$ in the absence of the the phase damping. At points $(p, t)=(0,0)$, the NLC measures have the values: $L(0)=U(0)=N(0)=0$ and $B(0)=1$ as we expected from the initial state of Equation (13).
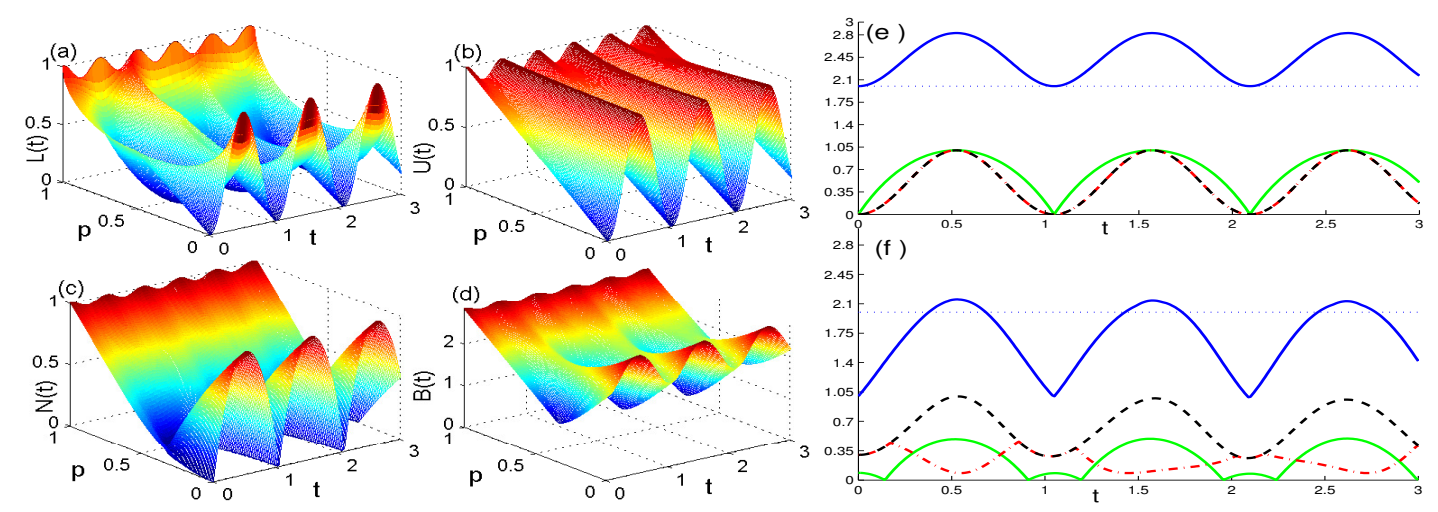

Figure 1. The 3D-evolution of $L(t)$ in (a), $U(t)$ in (b), $N(t)$ in (c) and $B(t)$ in (d) as functions of the time and the purity indicator $p \in[0,1]$ for $\chi_{z}=0.5, \Delta=1.5, J_{x}=J_{z}=2$ and $\gamma=0.0$. The 2D-evolution of $L(t)$ (dashed plots), $U(t)$ (dashed dotted plots), $B(t)$ (upper solid plots) and $N(t)$ (solid plots) for $p=0$ in (e) and $p=0.3$ in (f).

From Figure 1a-d, we observe that the evolutions of the NLC measures strongly depends on the purity indicator. The generated maximal and partial correlations of the LQU, the maximal Bell function and the log-negativity decrease for $p$ ranging from 0 to $p_{c}$ (critical points). After these critical points, they are enhanced with the increase of the purity indicator $p$. While the generated correlations of the UIN is enhanced monotonically with the increase of $p(p: 0 \rightarrow 1)$, without a critical point. Moreover, the generated maximal and partial correlations can be delayed/accelerated by particular choices for the purity of the initial states.

The two cases $p \in\left[0, p_{c}\right]$ and $p \in\left[p_{c}, 1\right]$ present opposite dynamical behavior for the NLCs. In the following, we investigate separately these two cases.

Case A: $p \in\left[0, p_{c}\right]$ In this interval $p \in\left[0, p_{c}\right]$, the generated partial and maximal correlations of the $\log$-negativity, Bell function, and LQU decrease as the purity indicator tends to the critical point $p_{c}$ $\left(p: 0 \rightarrow p_{c}\right)$. Here, we discuss the dynamics of the NLC for two cases: Pure state $p=0$ and partially corrected state $p=0.3$. Therefore, the 2D dynamics for the NLC functions $L(t), U(t), B(t)$, and $N(t)$ are shown in Figure 1e,f for these cases with the same parameters of the Figure 1a-d. From the 3D and 2D plots of Figure 1, we noted the following.

- The unitary DM interaction between the two qubits leads to the fact that the NLC functions present different generated correlations. Where the log-negativity, Bell function, LQU, and UIN correlations present different oscillatory behaviors. The generated maximal correlations depend on the purity indicator. In the case $p=0$, the NLC functions present partial and maximal correlations, whereas for the case $p=0.3$, the LQU generates a correlation greater than the other measures. The maximal violation of the Bell's inequality $(B(t)>2)$ depend on the purity indicator. In the case $p=0$, it occurs, whereas in the case $p=0.3$, it appears during small time intervals, in which the LQU and log-negativity correlations are stronger. As $p: 0 \rightarrow p_{c}$, the non-local correlation of $B(t)$ decreases. 
- The phenomenon of sudden changes appears only in the dynamics behavior of the log-negativity and the UIN correlations, represented by the abrupt changes in their dynamical behaviors. The phenomenon of the sudden changes was coined in [38] and experimentally observed in [39].

- During each period of the LQU and UIN behaviors, the UIN has two sudden change (SC) points. In the time intervals between the SC points, the dynamics of the LQU and UIN show maximal and minimal skew information correlations, respectively. While in the time intervals out sides these points, the LQU and UIN have the same correlation that is called the skew-information correlation (SI correlation) [12], it occurs at $L(t)=U(t))$ meaning that the minimal and maximal skew information correlations are equal.

- We have observed that, for $p=0.3$ and at some particular times, LQU and UIN present quantum correlations, whereas the system is disentangled and does not violates the Bell's inequality. It is worth noting that such similar observations were reported in $[40,41]$. The SI correlation depends on the purity indicator, where in the case $p=0$, it occurs during all the time, whereas in the case $p=0.3$, the SI correlation occurs in some time windows.

Figure 2 shows the effect of the DM interaction coupling $\chi_{z}=3.0$ on the dynamics behavior of the NLC functions $L(t), U(t), B(t)$ and $N(t)$. From 3D and 2D plots of Figure 2, we observe that the increase of the coupling of the DM interaction leads to more regular fluctuations, which increase as the purity indicator tends to the critical point $p_{c}\left(p: 0 \rightarrow p_{c}\right)$. The maxima of the $L(t)$ and $B(t)$ are points in their curves of the case $\chi_{z}=0.5$. The UIN correlation is enhanced by the DM interaction coupling. We note that the entanglement negativity does not change notably in this interval $p \in\left[0, p_{c}\right]$.
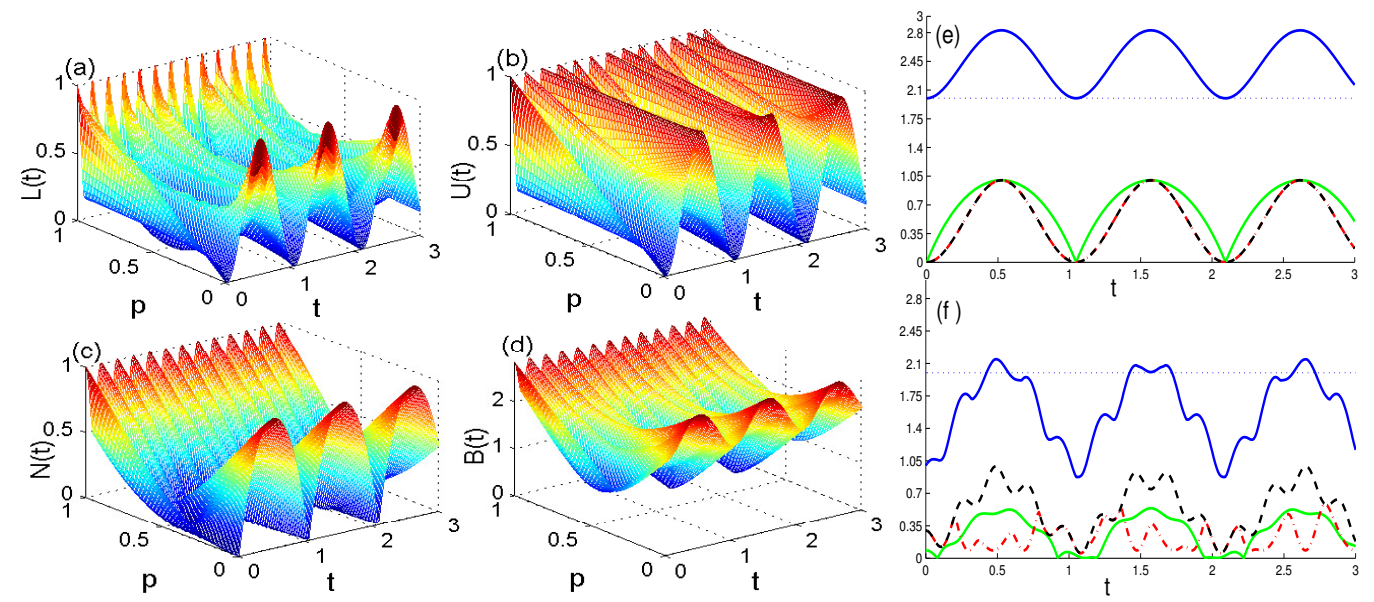

Figure 2. The $L(t)$ in (a), $U(t)$ in (b), $N(t)$ in (c) and $B(t)$ in (d) as functions of the time and the purity indicator $p \in[0,1]$ for $\chi_{z}=3, \Delta=1.5, J_{x}=J_{z}=2$ and $\gamma=0.0$. The $L(t)$ (dashed plots), $U(t)$ (dashed dotted plots), $B(t)$ (upper solid plots) and $N(t)$ (solid plots) for $p=0$ in (e) and $p=0.3$ in (f).

In Figure 3, the dependence of the NLC functions $L(t), U(t), B(t)$, and $N(t)$ on the anisotropy parameter $\Delta$, is plotted for the different cases: (1) In first case, the dynamics of the NLCs are shown for different values of the purity indicator $p \in[0,1]$ combined with the small value $\Delta=0.5$ in Figure $3 a, b$. (2) The NLCs are displayed for the anisotropy interval $\Delta \in[0,1.5]$ when the two qubits start with pure state in Figure $3 \mathrm{~g}-\mathrm{k}, p=0$. The 2D-NLC functions are plotted with the same data of Figure $3 \mathrm{a}, \mathrm{b}$, but for $p=0$ in Figure $3 \mathrm{e}$ and $p=0.3$ in Figure 3f. From Figure $3 \mathrm{a}, \mathrm{b}$, we note that the generated correlations, via the log-negativity, the maximal Bell function, and LQU, decrease with increasing $p: 0 \rightarrow p_{c}$. Meanwhile, the opposite behavior is observed for the generated UIN correlation, in other words, the UIN correlations is enhanced by the increase of the purity. The 2D plots show that, for small value of the anisotropy parameter, the period of the regular growth of the log-negativity, Bell function, LQU and UIN correlations grow with the same dynamical behavior. 

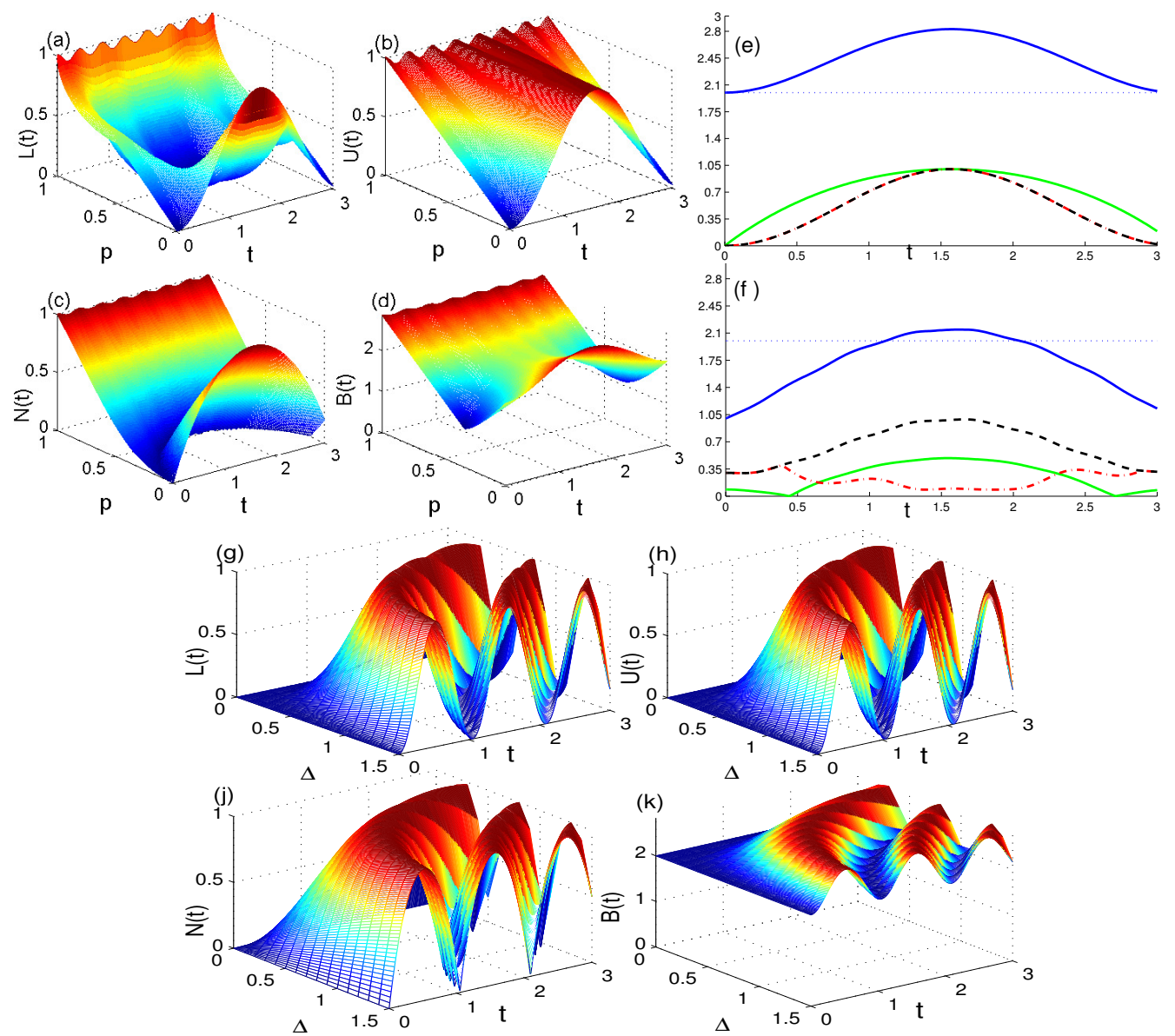

Figure 3. The 3D-evolution of $L(t)$ in (a), $U(t)$ in (b), $N(t)$ in (c) and $B(t)$ in (d) when $J_{x}=J_{z}=2$, $\chi_{z}=0.5$ and $\gamma=0$ for the cases: $p \in[0,1]$ with $\Delta=0.5$ in $(\mathrm{a}, \mathrm{b})$, and $\Delta \in[0,1.5]$ with $p=0$ in $(\mathbf{g}-\mathbf{k})$. The 2D-evolution of $L(t)$ (dashed plots), $U(t)$ (dashed dotted plots), $B(t)$ (upper solid plots) and $N(t)$ (solid plots) for $p=0$ in (e) and $p=0.3$ in (f).

Figure $3 \mathrm{~g}-\mathrm{k}$ reveals the effect of the anisotropy for the general choice $\Delta \in[0,1.5]$. As the anisotropy parameter increases, $\Delta: 0 \rightarrow 1.5$, the log-negativity, Bell function, and skew information quantifiers raise up (with respect of $\Delta$-axis) to their maximal correlations with oscillatory dynamics. The oscillations of the NLC functions along $\Delta$-axis increase by increasing time. The log-negativity reaches quickly its maximum than the others quantifiers.

In Figure 4, the effect of the phase damping parameter, $\gamma=0.4$, on the generated correlations is displayed with the same data in Figure 1. We observed that the phase damping leads to only reducing the amplitudes of the NLC functions with the same dynamical behavior. The entanglement log-negativity is more robust against the phase damping than the other NLCs.

The green solid curves of Figure $4 \mathrm{f}$, with the phase damping, show that the log-negativity $N(t)$ suddenly vanishes completely for a finite time and then it undergoes rebirth again. These phenomena of the sudden death and sudden birth of the entanglement [42,43] are observed only for the log-negativity entanglement for several finite periods (see the nest figure). Moreover, the phase damping $\gamma=0.4$ leads to increase of the partial SI correlation intervals and to the completely vanishing of the maximal violation of the Bell's inequality. For $p: 0 \rightarrow p_{c}$, the 3D plots of the Figure $4 \mathrm{a}-\mathrm{d}$ show that the purity indicator accelerates the effect of the the phase damping. 

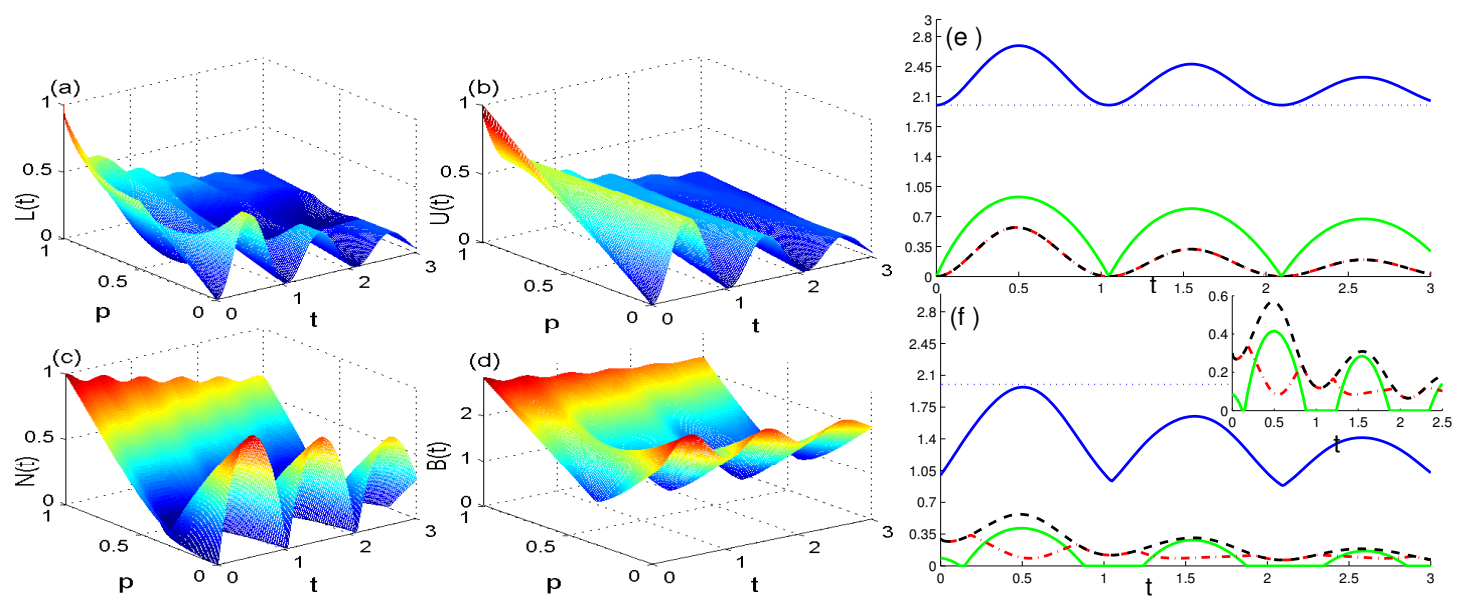

Figure 4. The 3D-evolution of $L(t)$ in (a), $U(t)$ in (b), $N(t)$ in (c) and $B(t)$ in (d) as functions of the time and the purity indicator $p \in[0,1]$ for $\chi_{z}=0.5, \Delta=1.5, J_{x}=J_{z}=2$ and $\gamma=0.4$. The 2D-evolution of $L(t)$ (dashed plots), $U(t)$ (dashed dotted plots), $B(t)$ (upper solid plots) and $N(t)$ (solid plots) for $p=0$ in (e) and $p=0.3$ in (f).

In Figure 5, the dynamics for the NLC functions $L(t), U(t), B(t)$ and $N(t)$ are shown for the case $p=0.5$. The maximal violation of the Bell's inequality $(B(t)>2)$ disappears completely. The $B(t)$ has oscillometry behavior depending on the parameters of the anisotropy, the coupling to the of DM interaction, and the coupling environment. Figure $5 \mathrm{~b}$ confirms that the DM interaction coupling leads to more regular oscillations, while Figure $5 \mathrm{c}$ shows that the anisotropy parameter control the period of the growth of the correlation functions. For the case $p=0.5$, the deterioration of the NLCs, and the sudden death and sudden birth phenomena are more pronounced than the others cases $p=0$ and $p=0.3$.
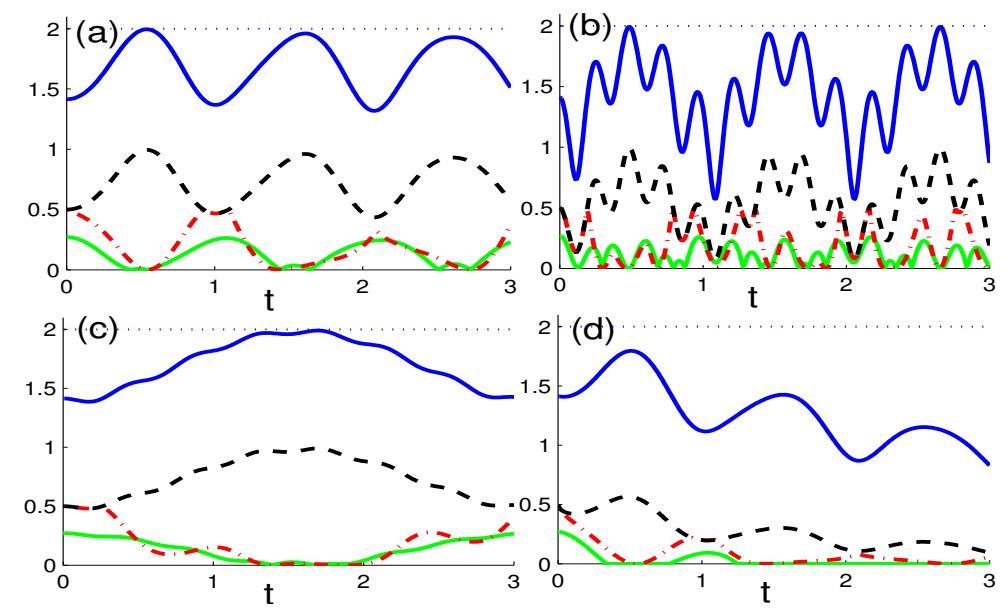

Figure 5. The $L(t)$ (dashed plots), $U(t)$ (dashed dotted plots), $B(t)$ (upper solid plots), and $N(t)$ (solid plots) for $J_{x}=J_{z}=2$ and $p=0.5$ with the cases $\left(\chi_{z}, \Delta, \gamma\right)=(0.5,1.5,0.0)$ in $(\mathbf{a}) ;\left(\chi_{z}, \Delta, \gamma\right)=(3$, $1.5,0.0)$ in $(\mathbf{b}) ;\left(\chi_{z}, \Delta, \gamma\right)=(0.5,0.5,0.0)$ in $(\mathbf{c})$ and $\left(\chi_{z}, \Delta, \gamma\right)=(0.5,1.5,0.4)$ in (d).

Case B: $p \in\left[p_{c}, 1\right]$.

In this subsection, the non-classical correlations are explored for the purity indicator between $p_{c}$ and $1, p \in\left[p_{c}, 1\right]$. From Figures $1,4 \mathrm{a}-\mathrm{d}, 6$ and 7 , we conclude that the NLCs grow with the increasing of the purity indicator $p$. This behavior is opposite to what has been observed for $p$ between 0 and $p_{c}$. 

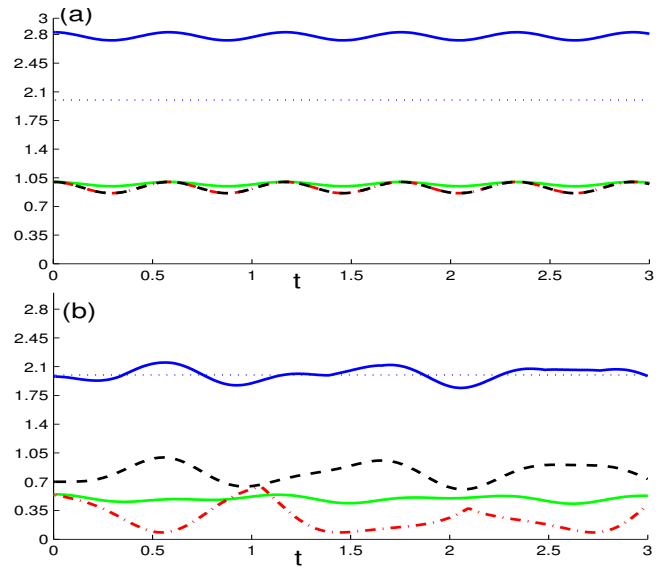
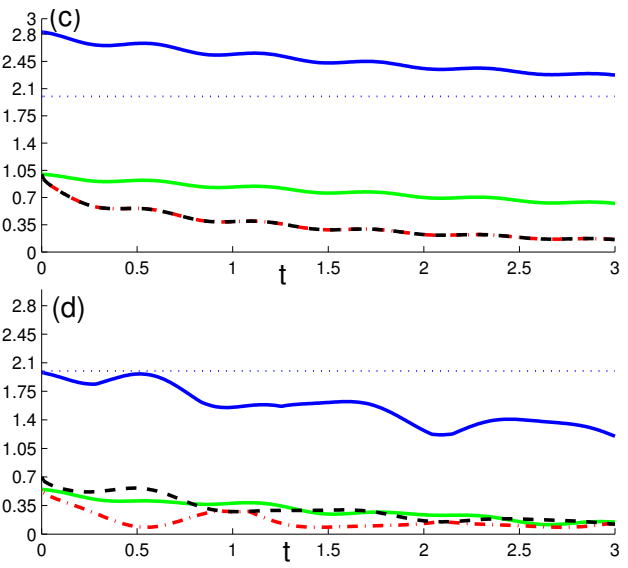

Figure 6. The $L(t)$ (dashed plots), $U(t)$ (dashed dotted plots), $B(t)$ (upper solid plots), and $N(t)$ (solid plots) for $\chi_{z}=0.5, \Delta=1.5, p=1, J_{x}=J_{z}=2$ and $\gamma=0.0$ for: $p=1$ in panel (a) and $p=0.7$ in panel (b). While in panels (c,d) $\gamma=0.4$.

From 3D plots of Figure 1 and 2D plots of Figure 6a,b, we notice that: (1) For $p=1$ and $\gamma=0$, the effect of the unitary interaction on the NLC evolutions is very weak compared to the case $p=0.7$. As the purity indicator decreases , $p: 1 \rightarrow p_{c}$, the unitary interaction effect raise up, and the initial NLCs is deteriorated, except the maximal correlation of the UIN. (2) The maximal violation of the Bell's inequality and SI correlations appear for $p=1$. While for $p=0.7$, the SI correlation disappears, the violation of $B(t)$ is reduced, and the phenomenon of sudden changes manifests only in the dynamics of the UIN.
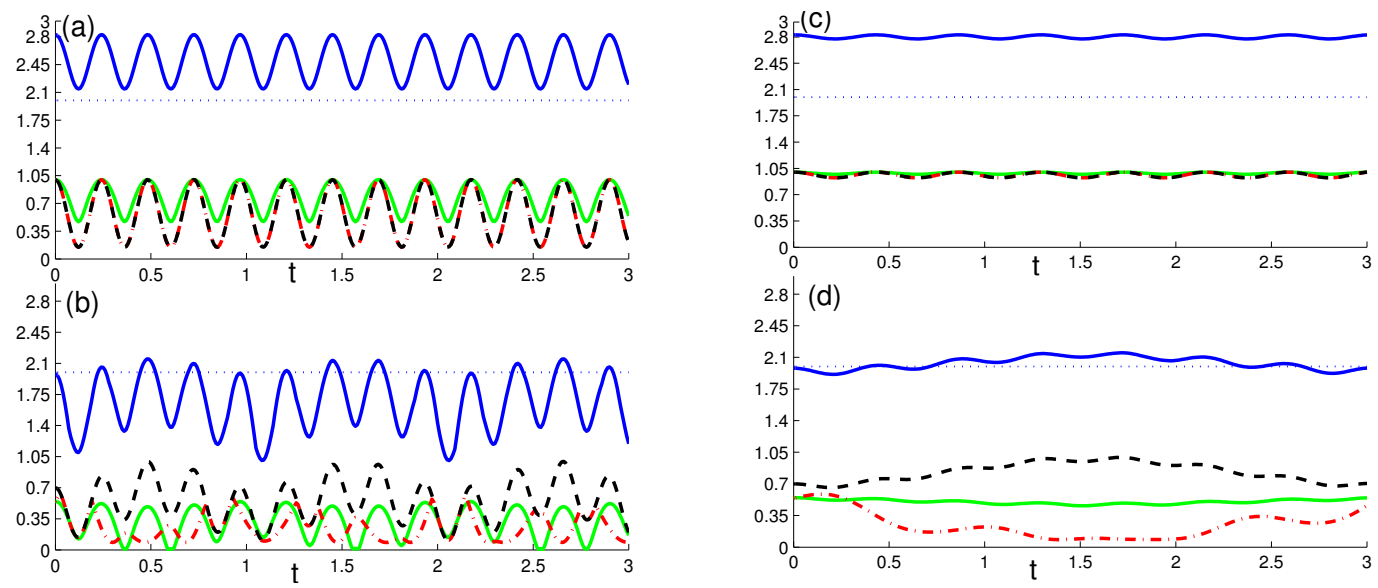

Figure 7. As Figure $6 \mathrm{a}, \mathrm{b}$ but for $\chi_{z}=3$ in panels $(\mathbf{a}, \mathbf{b})$ and $\Delta=0.5$ in panels $(\mathbf{c}, \mathbf{d})$.

Figure $6 c$,d shows the effects of the phase damping $\gamma=0.4$ on the the initial maximal $(p=1)$ and partial $(p=0.7)$ NLCs. The NLC amplitudes are reduced when the time evolves. As observed from Figure $6 \mathrm{a}-\mathrm{d}$, the deterioration of the NLCs increases as $p: 1 \rightarrow p_{c}$. The NLC quantifiers are more sensitive to the phase damping, and the log-negativity entanglement is more robust against the phase damping.

From Figures $2 \mathrm{a}-\mathrm{d}$ and $7 \mathrm{a}, \mathrm{b}$ we observe that a lager value of the DM interaction $\chi_{z}=3$ leads to the enhancement of the effect of the unitary interaction. In the range $p \in\left[p_{c}, 1\right]$, the DM interaction has strong effect unlike the case $p \in\left[0, p_{c}\right]$. The NLCs for $p$ ranging from $p_{c}$ to 1 is plotted in the Figure $3 \mathrm{a}-\mathrm{d}$ with large value $\Delta=1.5$. While Figure $7 \mathrm{c}$, d represents the NLCs for a small value of the anisotropy, $\Delta=0.5$, with $p=1$ and $p=0.7$. For the case $p=1$, the NLCs have small oscillations with weak amplitudes, while for the case $p=0.7$, they have notable changes, see Figure $7 \mathrm{c}$,d. The correlations of the LQU and the Bell's inequality are more affected than the others quantifiers. 
The two-qubit Heisenberg XYZ system can be realized from spin-1/2 pyrochlores R2V2O7 [30]. Our results present several potential applications in quantum processing as generation of quantum correlations $[27,28]$ and quantum simulations [44]. Quantum simulations are required to solve various complex problems in diverse areas of science such as exploring complex molecular structure in chemistry [45], studying disorder systems in many-body physics [46], and conceiving high-temperature superconductor materials $[47,48]$. The two-qubit entangled system can be used to transfer unknown quantum state by employing quantum teleportation [49], or classical information through quantum dense coding [50], as the degree of entanglement plays a vital role to transfer the unknown quantum state in a perfect or probabilistic way. Furthermore, it could manage the transmission capability of the classical information, which is a crucial parameter to estimate the quality of performing dense coding. Finally, the approach used here can be applied to other physical systems. The results could be important for quantum optics, quantum computation, and quantum machine learning.

\section{Conclusions}

We have studied the nonlocal correlation dynamics of two qubits in anisotropic Heisenberg model with Dzyaloshinskii-Moriya interaction via the log-negativity, Bell function, local quantum uncertainty, and uncertainty-induced non-locality. In our investigation, we consider the coupling of DM interaction, the coupling to the environment, and the anisotropy. When the system is initially prepared in the class of mixed Werner state, it is found that the purity indicator plays a key role for the nonlocal correlations. These correlations also depend on the DM interaction, the anisotropy, and the phase damping. It is observed that both the log-negativity and the UIN correlations exhibit sudden-change behavior. Furthermore, the system presents skew-information correlation. We have deduced that the purity indicator, the DM interaction, the anisotropy, and the phase damping control the SI correlation, the Bell's inequality correlation, and the sudden death and sudden birth of the entanglement.

Author Contributions: The all authors contributed equally to the manuscript. All authors have read and agreed to the published version of the manuscript.

Funding: This project was supported by the Deanship of Scientific Research at Prince Sattam Bin Abdulaziz University under the research project No. 2020/01/11801.

Acknowledgments: The authors are very grateful to the referees for their constructive remarks which have helped to improve the manuscript.

Conflicts of Interest: The authors declare no conflict of interest.

\section{References}

1. Nielsen, M.A.; Chuang, I.L. Quantum Computation and Quantum Information; Cambridge University Press: Cambridge, UK, 2000.

2. Abliz, A.; Gao, H.J.; Xie, X.C.; Wu, Y.S.; Liu, W.M. Entanglement control in an anisotropic two-qubit Heisenberg X Y Z model with external magnetic fields. Phys. Rev. A 2006, 74, 052105. [CrossRef]

3. Zhang, Y.; Zhou, Q.; Xu, H.; Fang, M. Quantum-Memory-Assisted Entropic Uncertainty in Two-Qubit Heisenberg XX Spin Chain Model. Int. J. Theor. Phys. 2019, 58, 4194. [CrossRef]

4. Sete, E.A.; Eleuch, H. High-efficiency quantum state transfer and quantum memory using a mechanical oscillator. Phys. Rev. A 2015, 91, 032309. [CrossRef]

5. Mohamed, A.-B.; Eleuch, H. Non-classical effects in cavity QED containing a nonlinear optical medium and a quantum well: Entanglement and non-Gaussanity. Eur. Phys. 2015, 69, 191. [CrossRef]

6. Luo, S. Using measurement-induced disturbance to characterize correlations as classical or quantum. Phys. Rev. A 2008, 77, 022301. [CrossRef]

7. Ollivier, H.; Zurek, W.H. Quantum Discord: A Measure of the Quantumness of Correlations. Phys. Rev. Lett. 2001, 88, 017901. [CrossRef]

8. Henderson, L.; Vedral, V. Classical, quantum and total correlations. J. Phys. A 2001, 34, 6899. [CrossRef]

9. Luo, S.; Fu, S. Measurement-Induced Nonlocality. Phys. Rev. Lett. 2011, 106, 120401. [CrossRef] 
10. Wigner, E.P.; Yanase, M.M. Information Contents of Distributions. Proc. Natl. Acad. Sci. USA 1963, 49, 910. [CrossRef]

11. Paula, F.M.; de Oliveira, T.R.; Sarandy, M.S. Geometric quantum discord through the Schatten 1-norm. Phys. Rev. A 2013, 87, 064101. [CrossRef]

12. Mohamed, A.-B.A.; Eleuch, H. Generation and robustness of bipartite non-classical correlations in two nonlinear microcavities coupled by an optical fiber. J. Opt. Soc. Am. B 2018, 35, 47. [CrossRef]

13. Tacchino, F.; Auffèves, A.; Santos, M.F.; Gerace, D. Steady State Entanglement beyond Thermal Limits. Phys. Rev. Lett. 2018, 120, 063604. [CrossRef] [PubMed]

14. Piani, M. Problem with geometric discord. Phys. Rev. A 2012, 86, 034101. [CrossRef]

15. Girolami, D.; Tufarelli, T.; Adesso, G. Characterizing Nonclassical Correlations via Local Quantum Uncertainty. Phys. Rev. Lett. 2013, 110, 240402. [CrossRef] [PubMed]

16. Wu, S.-X.; Zhang, J.; Yu, C.-S.; Song, H.-S. Uncertainty-induced quantum nonlocality. Phys. Lett. A 2014, 378, 344. [CrossRef]

17. Giulini, D.; Joos, E.; Kiefer, C.; Kupsch, J.; Stamatescu, I.-O.; Zeh, H.D. Decoherence and the Appearance of a Classical World; Springer: Berlin, Germany, 1996.

18. Obada, A.-S.F.; Hessian, H.A.; Mohamed, A.-B.A. Entropies and entanglement for decoherence without energy relaxation in a two-level atom. J. Phys. B At. Mol. Opt. Phys. 2007, 40, 2241. [CrossRef]

19. Duan, L.-M.; Guo, G.-C. Preserving Coherence in Quantum Computation by Pairing Quantum Bits. Phys. Rev. Lett. 1997, 79, 1953. [CrossRef]

20. Pourkarimi, M.R.; Rahnama, M.; Rooholamin, H. Decoherence effect on quantum correlation and entanglement in a two-qubit spin chain. In. J. Theor. Phys. 2015, 54, 1085. [CrossRef]

21. Zhang, Y.; Zhou, Q.; Fang, M.; Kang, G.; Li, X. Quantum-memory-assisted entropic uncertainty in two-qubit Heisenberg XYZ chain with Dzyaloshinskii-Moriya interactions and effects of intrinsic decoherence. Quantum Inf. Process. 2018, 17, 326. [CrossRef]

22. Loss, D.; DiVincenzo, D.P. Quantum computation with quantum dots. Phys. Rev. A 1998, 57, 120. [CrossRef]

23. Khitrova, G.; Gibbs, H.M.; Kira, M.; Koch, S.W.; Scherer, A. Vacuum Rabi splitting in semiconductors. Nat. Phys. 2006, 2, 81.

24. Dzyaloshinsky, I. A thermodynamic theory of weak ferromagnetism of antiferromagnetics. J Phys. Chem. Solids 1958, 4, 241.

25. Moriya, T. Anisotropic Superexchange Interaction and Weak Ferromagnetism. Phys. Rev. 1960, $120,91$. [CrossRef]

26. Radhakrishnan, C.; Parthasarathy, M.; Jambulingam1, S.; Byrnes, T. Quantum coherence of the Heisenberg spin models with Dzyaloshinsky-Moriya interactions. Sci. Rep. 2017, 7, 13865.

27. Qin, M.; Ren, Z.-Z. Influence of intrinsic decoherence on entanglement teleportation via a Heisenberg XYZ model with different Dzyaloshinskii-Moriya interactions. Quantum Inf. Process. 2015, 14, 2055. [CrossRef]

28. Sun, Y.; Ma, X.-P.; Guo, J.-L. Dynamics of non-equilibrium thermal quantum correlation in a two-qubit Heisenberg XYZ model. Quantum Inf. Process. 2020, 19, 98. [CrossRef]

29. Mohamed, A.-B.A.; Eleuch, H. Quantum correlation control for two semiconductor microcavities connected by an optical fiber. Phys. Scr. 2017, 92, 065101.

30. Xiang, H.J.; Kan, E.J.; Whangbo, M.-H.; Lee, C.; Wei, S.; Gong, X.G. Single-ion anisotropy, DzyaloshinskiiMoriya interaction, and negative magnetoresistance of the spin- $\frac{1}{2}$ pyrochlore $R_{2} V_{2} O_{7}$. Phys. Rev. B 2011, 83, 174402. [CrossRef]

31. Hessian, H.A.; Obada, A.-S.F.; Mohamed, A.-B.A. Effect of atomic spontaneous decay on entanglement in the generalized Jaynes-Cummings model. Ann. Phys. 2010, 325 519. [CrossRef]

32. Mohamed, A.-B.A.; Eleuch, H.; Raymond Ooi, C. H. Non-locality Correlation in Two Driven Qubits Inside an Open Coherent Cavity: Trace Norm Distance and Maximum Bell Function. Sci. Rep. 2019, 9, 19632. [CrossRef]

33. Caminati, M.; Martini, F.D.; Perris, R.; Sciarrino, F.; Secondi, V. Nonseparable Werner states in spontaneous parametric down-conversion. Phys. Rev. A 2006, 73, 032312.

34. Barbieri, M.; Martini, F.D.; Nepi, G.D.; Mataloni, P. Generation and Characterization of Werner States and Maximally Entangled Mixed States by a Universal Source of Entanglement. Phys. Rev. Lett. 2004, 92, 177901. [CrossRef] [PubMed] 
35. Kumano, H.; Matsuda, K.; Ekuni, S.; Sasakura, H.; Suemune, I. Characterization of two-photon polarization mixed states generated from entangled-classical hybrid photon source. Optics Express 2011, 19, 14249. [CrossRef] [PubMed]

36. Vidal, G.; Werner, R.F. Computable measure of entanglement. Phys. Rev. A 2002, 65, 032314. [CrossRef]

37. Horodecki, R.; Horodecki, P.; Horodecki, M. Violating Bell inequality by mixed spin- $\frac{1}{2}$ states: Necessary and sufficient condition. Phys. Lett. A 1995, 200, 340.

38. Maziero, J.; Celeri, L.C.; Serra, R.M.; Vedral, V. Classical and quantum correlations under decoherence. Phys. Rev. A 2009, 80, 044102.

39. Xu, J.-S.; Xu, X.-Y.; Li, C.-F.; Zhang, C.-J.; Zou, X.-B.; Guo, G.-C. Experimental investigation of classical and quantum correlations under decoherence. Nat. Commun. 2010, 1, 7.

40. Streltsov, A.; Adesso, G.; Plenio, M.B. Quantum coherence as a resource. Rev. Mod. Phys. 2017, 89, 041003. [CrossRef]

41. We, T.-C.; Nemoto, K.; Goldbart, P.M.; Kwiat, P.G.; Munro, W.J.; Verstraete, F. Maximal entanglement versus entropy for mixed quantum states. Phys. Rev. A 2003, 67, 022110. [CrossRef]

42. Yu, T.; Eberly, J.H. Finite-Time Disentanglement Via Spontaneous Emission. Phys. Rev. Lett. 2004, $93,140404$.

43. Yu, T.; Eberly, J.H. Sudden Death of Entanglement. Science 2009, 323, 598. [CrossRef] [PubMed]

44. Salathé, Y.; Mondal, M.; Oppliger, M.; Heinsoo, J.; Kurpiers, P.; Potočnik, A.; Mezzacapo, A.; Heras, U.L.; Lamata, L.; Solano, E.; et al. Digital Quantum Simulation of Spin Models with Circuit Quantum Electrodynamics. Phys. Rev. X 2015, 5, 021027.

45. Lu, D.; Xu, B.; Xu, N.; Li, Z.; Chen, H.; Peng, X.; Xu, R.; Du, J.-F. Quantum chemistry simulation on quantum computers: Theories and experiments. Phys. Chem. Chem. Phys. 2012, 14, 9411. [CrossRef] [PubMed]

46. Choi, J.-Y.; Hild, S.; Zeiher, J.; Schauß, P.; Rubio-Abadal, A.; Yefsah, T.; Khemani, V.; Huse, D.A.; Bloch, I.; Gross, C. Exploring the many-body localization transition in two dimensions. Science 2016, 352, 1547.

47. Mazurenko, A.; Chiu, C.S.; Ji, G.; Parsons, M.F.; Kanász-Nagy, M.; Schmidt, R.; Grusdt, F.; Demler, E.; Greif, D.; Greiner, M. A cold-atom Fermi-Hubbard antiferromagnet. Nature 2017, 545, 462. [CrossRef]

48. Obada, A.-S.F.; Hessian, H.A.; Mohamed, A.-B.A.; Homid, A.H. Implementing discrete quantum Fourier transform via superconducting qubits coupled to a superconducting cavity. J. Opt. Soc. Am. B 2013, $30,1178$.

49. Xu, H.; Yong, L.; Liao, S.; Yin, J.; Cai, W.; Yang, M.; Li, L.; Yang, K.; Han, X.; Yao, Y.; et al. Ground-to-satellite quantum teleportation. Nature 2017, 549, 70.

50. Nielsen, M.A.; Chuang, I.L. Application: Superdense coding. Quantum Computation and Quantum Information, 10th ed.; Cambridge University Press: Cambridge, UK, 2010.

(C) 2020 by the authors. Licensee MDPI, Basel, Switzerland. This article is an open access article distributed under the terms and conditions of the Creative Commons Attribution (CC BY) license (http://creativecommons.org/licenses/by/4.0/). 